\title{
Noch mehr Kompetenzen für Verfassungsschutz?
}

Man könnte meinen, wir leben in den USA: Da produzierten Geheimdienste - das Bundesamt für Verfassungsschutz (BfV) und dessen kleine Brüder in den Ländern - beim Umgang mit dem Nationalsozialistischen Untergrund (NSU) tödliche Fehler. Parlamentarische Untersuchungen kommen zum Ergebnis, dass diese auf nicht genutzten Befugnissen und zu wenig Kontrolle beruhen. Und dann werden diesem BfV weitere Befugnisse zugesprochen und die Kontrolldefizite bleiben bestehen. Nach grundsätzlicher Zustimmung der Bundesländer in der Innenministerkonferenz im Dezember 2014 legte die Bundesregierung am 27.03.2015 einen Gesetzentwurf vor, den sie im Schnelldurchgang und im Windschatten der Diskussion um die Vorratsspeicherung von Telekommunikationsverkehrsdaten durchzuziehen versucht.

Der Entwurf für ein „Gesetz zur Verbesserung der Zusammenarbeit im Bereich des Verfassungsschutzes“ (BR-Drs. 123/15), dessen erklärtes Ziel darin besteht, aus den NSU-Ermittlungspannen rechtliche Konsequenzen zu ziehen, greift nicht die wesentlichen Vorschläge des NSU-Untersuchungsausschusses zur Verbesserung der Geheimdienstkontrolle auf, sondern erleichtert die $\mathrm{Da}$ tenerhebung und den Datenaustausch, ohne datenschutzrechtliche, parlamentarische oder sonstige rechtsstaatliche Vorkehrungen einzubauen.

Der Entwurf sieht u. a. Folgendes vor:

- Das BfV erhält umfassende Ermittlungskompetenzen in den Ländern, ohne mit dem Landesverfassungsschutz Einvernehmen herstellen zu müssen, so dass weiterhin Parallel- und Konkurrenzermittlungen stattfinden können.

- Das BfV erhält eine umfassende Auswertekompetenz zu „allen Erkenntnissen über Bestrebungen“ des Verfassungsschutzes der Länder.

- Mit einer Regelung zur technischen „Unterstützung“ der Länder durch das BfV soll - mit einer untauglichen Regelung - die bisher unzulässige Form des BfV-Hostings für die Länder legalisiert werden.

- Die Verfassungsschutzämter der Länder werden zur Datenübermittlung verpflichtet bei „Relevanz“ statt „Erforderlichkeit“, womit eine verfassungsrechtlich unzulässige Senkung der Schwelle des Eingriffs in das Grundrecht auf informationelle Selbstbestimmung verbunden ist.

- Die technisch-organisatorischen Schutzvorkehrungen des gemeinsam von den Verfassungsschutzämtern betriebenen Systems NADIS-Wissensnetz (NADIS-WN) mit hochsensiblen Daten sind völlig unzureichend.

- Polizeien und Staatsanwaltschaften der Länder werden - unter Ausblendung des verfassungsrechtlich begründeten Trennungsgebots - zur Datenübermittlung an das BfV verpflichtet.
Die Gesamtheit dieser Neuregelungen laufen darauf hinaus, dass das BfV in NSA-Manier Big-Data-Analysen sämtlicher beim Verfassungsschutz vorhandener Daten durchführen können soll.

Weiterhin ist vorgesehen, dass V-Leute und verdeckte Ermittler des Verfassungsschutzes in Zukunft straffrei Straftaten wie Körperverletzungen, Erpressung, Drogenhandel oder unerlaubten Waffenbesitz begehen können. Damit wird der Graubereich bei Ermittlungen im extremistischen Milieu nicht aufgehellt, sondern verdunkelt. Hintertüren für die Anwerbung und den Einsatz werden eher geöffnet als geschlossen. Die vorgesehenen Eingrenzungen bei den sog. Vertrauenspersonen sind bei genauer Analyse wenig effektiv.

Die Konferenz der Datenschutzbeauftragten des Bundes und der Länder hat mit einer Entschließung zur Geheimdienstkontrolle im Oktober 2014 konkrete Vorschläge gemacht, wie die bestehenden Kontrolldefizite unter umfassender Einbeziehung des Datenschutzes abgebaut werden können. Aus Grundrechtssicht ist es geradezu absurd, dass in diesem Entwurf wie auch z. B. im zeitgleich im Parlament verhandelten IT-Sicherheitsgesetz der Verfassungsschutz personell massiv ausgebaut werden soll, während der Datenschutz praktisch leer ausgeht. Parallel zum Datenschutz sollte ein Geheimdienstbeauftragter eingerichtet werden, der mit eigenem Personal und im parlamentarischen Auftrag die laufenden Geschäfte der Dienste auf ihre Rechtsstaatlichkeit hin hinterfragt. Doch davon steht im Gesetzentwurf nichts.

Der Gesetzentwurf stammt aus der Feder von denjenigen, die ein Teil des zu lösenden Problems sind: der Verfassungsschutzbürokratie des Bundes. Der Entwurf ist unausgegoren und bedarf der öffentlichen Debatte, weil er die NSU-Probleme nicht löst, sondern verschärft. Die öffentliche Aufmerksamkeit im Sicherheitsbereich konzentriert sich derzeit auf die TK-Vorratsdatenspeicherung. So wichtig diese Diskussion ist, dürfen in deren Windschatten nicht Gesetze wie das vorliegende durchgewunken werden, die die Vergeheimdienstlichung unserer Gesellschaft vorantreiben.

Thilo Weichert, Unabhängiges Landeszentrum für Datenschutz Schleswig-Holstein (ULD)

1 Stellungnahmen zum Gesetzentwurf finden sich unter https://www.datenschutzzentrum.de/artikel/895-.html (ULD) https://netzpolitik.org/2015/verfassungsschutz-gesetz-die-stellungnahmeder-datenschutzbeauftragten-die-der-union-zu-kritisch-war/\#StellungnahmeBfDI (BfDI) 